\title{
Democracia delegativa en Argentina, Presidencia 2015-2019: Antiguas democracias y república moderna
}

Delegative democracy in Argentina, Presidency 2015-2019: Old democracies and modern republic Democracias delegativas na Argentina, Presidência 2015-2019: Velhas democracias e república moderna

\section{Paula Daniela Franco}

paula.franco@yahoo.com.ar

Universidad de Buenos Aires (UBA) - Argentina

ORCID: https://orcid.org/0000-0001-6693-7929

\begin{abstract}
RESUMEN
La única presidencia del Ingeniero civil Mauricio Macri fue elegida por el período de 4 años desde diciembre del 2015 al 2019 con el partido político Cambiemos en la República Argentina gracias al sistema democrático y representativo de dicho país. Resulta interesante analizarlo como un caso de ejemplo en donde las democracias delegativas surgen de las crisis. La experiencia de Cambiemos marca el inicio de una nueva e incierta transición en la Argentina, no ya de gobierno sino de régimen democrático, de la democracia delegativa a una auténtica democracia representativa y republicana. En ese sentido, delimitar las diferencias entre las antiguas democracias y la república resulta necesario para comprender la coyuntura actual en el país latinoamericano.
\end{abstract}

Palabras clave/descriptores: Democracia. Argentina. Política. Estado de derecho

\begin{abstract}
The only presidency of the civil engineer Mauricio Macri was elected for the period of 4 years from December 2015 to 2019 with the political party Cambiemos in the Argentine Republic, thanks to the democratic and representative system of that country. It is interesting to analyze it as an example case where delegative democracy arises from crises. The experience of Cambiemos marks the beginning of a new and uncertain transition in Argentina, not just from government but from democratic regime, from delegative democracy to an authentic representative and republican democracy. In this sense, delimiting the differences between the old democracies and the republic is necessary to understand the current situation in the Latin American country.
\end{abstract}

Keywords: Democracy. Argentina. Politics. Rule of law

\section{RESUMO}

A única presidência do engenheiro civil Mauricio Macri foi eleita para o período de 4 anos de dezembro de 2015 a 2019 com o partido político Mudança na República Argentina graças ao sistema democrático e representativo de dito país. É interessante analisá-lo como um exemplo de caso em que a democracia delegativa surge de crises. A experiência de Cambiemos marca o início de uma nova e incerta transição na Argentina, não apenas do governo, mas do regime democrático, da democracia delegativa a uma autêntica democracia representativa e republicana. Nesse sentido, delimitar as diferenças entre as velhas democracias e a república é necessário para entender a situação atual do país latino-americano.

Palavras-chave: Democracia. Argentina. Política. Estado de Direito 


\section{Introducción}

A lo largo de los siglos, el concepto de democracia se fue profundizando y analizando a través de la mirada de diversos autores y académicos. El impacto de las tradiciones liberales y republicanas en el desarrollo conceptual e institucional de la democracia representativa moderna ha influido en la toma de decisiones de los ciudadanos.

En primer lugar, es importante plantear un desarrollo teórico que acuña qué se entiende por gobierno democrático planteando un breve recorrido social, histórico y contextual. Por otro lado, desarrollar cuáles son las principales diferencias entre las ideas de las antiguas democracias y las repúblicas modernas sirve para repensar las condiciones y sucesos que se viven hoy en día.

En ese sentido, se tomará como ejemplo el caso de la única presidencia del Ingeniero Mauricio Macri, quien asumió en el año 2015 hasta diciembre del 2019. Es elegido a través del sistema democrático y representativo como presidente estando al frente del partido político Cambiemos en la República Argentina. Su mandato sirvió para analizar cómo se percibe el Estado de Derecho en el Siglo XXI, la democracia representativa, la accountability horizontal y el rol del liderazgo en los actores políticos democráticos.

\section{Desarrollo teórico}

Los gobiernos democráticos contemporáneos han evolucionado a partir de un sistema político que fue concebido por sus fundadores en oposición a la democracia (Manin, 2006). El término "democracia" en su sentido jurídico-institucional y no en su significado ético, o sea, en un sentido más procesal que sustancial.

Es indudable que históricamente "democracia" tiene dos significados preponderantes, por lo menos en su origen, según sí pone en mayor evidencia el conjunto de reglas cuya observancia es necesaria con objeto de que el poder político sea distribuido efectivamente entre la mayor parte de los ciudadanos, las llamadas reglas del juego, o el ideal en el cual un gobierno democrático debería inspirarse, que es el de la igualdad. (Bobbio, 2005, p.6).

Como también considera Dahl (1999) postulando que un avance hacia la participación democrática se desarrolla desde lo que se denomina la lógica de la igualdad. En otras palabras, la democracia se vincula a la institucionalización y vigencia de derechos políticos de participación y deliberación siendo el más importante la participación del voto para llegar a una mayor igualdad. Dahl plantea que la democracia es fundamental para que la sociedad tenga un desarrollo social, cultural, político y pueda consolidarse.

Como también considera Dahl (1999) postulando que un avance hacia la participación democrática se desarrolla desde lo que se denomina la lógica de la igualdad. En otras palabras, la democracia se vincula a la institucionalización y vigencia de derechos políticos de participación y deliberación siendo el más importante la participación del voto para llegar a una mayor igualdad. Dahl plantea que la democracia es fundamental para que la sociedad tenga un desarrollo social, cultural, político y pueda consolidarse. 
"La democracia puede ser inventada y reinventada de manera independiente dondequiera que se den las condiciones adecuadas. $\mathrm{Y}$ las condiciones adecuadas han existido en tiempos distintos y en lugares diferentes" (Dahl, 1999, p.12). Desarrolla el concepto de poliarquía como conjunto de reglas que designan cómo se llega al poder, quiénes llegan y cómo se ejerce. Tiene un conjunto de características y se corresponde con una manifestación empírica de las democracias contemporáneas.

Es importante destacar que la democracia como se viene mencionando, y la república se dividen en un momento específico en el cual se institucionaliza la república moderna como forma de gobierno representativo. Siéyès consideraba sobre todo la representación como la aplicación en el ámbito político de la división del trabajo, un principio que, en su opinión, constituye un factor clave del progreso social.

En relación a los impactos las tradiciones liberales y republicanas en el desarrollo conceptual de las instituciones de las democracias representativas modernas se puede hacer alusión a Ortiz Leroux (2014) porque considera que con el renacimiento de las ciudades luego de la Edad Media, el republicanismo ha tenido también un renacer, es decir, que el mismo no fue el resultado de la casualidad ni de una supuesta voluntad divina o ley histórica, sino el resultado, principalmente de un vacío o malestar que fue diagnosticado en el cuerpo político de las sociedades liberales contemporáneas. Es interesante considerar los aportes tanto de Roma como de Grecia Antigua en la constitución de lo que luego se dio a llamar democracias representativas.

En efecto, el Renacimiento de la teoría política republicana se ve relacionado a una crisis de doble entrada: por una parte, una crisis política ligada a la falta de representatividad y legalidad de las democracias liberales existentes, las cuales ha sustituido la figura política del ciudadano por una imagen económica del consumidor y "han relegado las virtudes cívicas clásicas por las virtudes institucionales modernas, y por otra parte una crisis teórica ligada a la fragilidad de los fundamentos normativos del liberalismo conservador" (Ortiz Leroux, 2014, p.24).

Por ejemplo, según Manin (2006) las repúblicas italianas de la Edad Media y del Renacimiento elegían a menudo sus magistrados por sorteo. En Florencia, centro intelectual del humanismo cívico y de la renovación republicana, la selección de magistrados por sorteo era una institución clave del sistema republicano.

El politólogo estadounidense, Dahl (1999), considera que la democracia es antigua y se pregunta “¿dónde se inició exactamente?”, algunos pueden pensar que la democracia comenzó hace doscientos años en los Estados Unidos, otros en la Grecia Antigua o Roma. La idea de una continuidad de la democracia lineal es una premisa que Dahl pone en cuestión por dos motivos. En primer lugar, porque después de los primeros siglos en Grecia y Roma, el progreso del gobierno popular derivó en su caída y desaparición. En segundo lugar, la democracia no fue inventada de una vez y para siempre. Aunque Roma, según Dahl (1999) ha sido un pueblo altamente creativo, nunca inventaron o adaptaron una solución que hoy no parece obvia: un sistema factible de gobierno representativo.

Para los Federalistas, "la constitución de una república moderna debe ser fundada en un conocimiento preciso de las consecuencias que tienen las leyes y de las instituciones en el comportamiento humano" (Ortiz Leroux, 2014, p.45). En palabras del autor, desde esta perspectiva 
las leyes e instituciones son las que aseguran la existencia y durabilidad de las repúblicas y no las cualidades cívicas de los ciudadanos.

La verdadera diferencia entre las antiguas democracias y las repúblicas modernas estriba, de acuerdo con Madison, en la «la absoluta exclusión del pueblo en su calidad de colectivo de cualquier participación en el gobierno en las segundas, y no en la absoluta exclusión de los representantes del pueblo de la administración en las primeras».

"La democracia puede ser inventada y reinventada de manera independiente dondequiera que se den las condiciones adecuadas. $\mathrm{Y}$ las condiciones adecuadas han existido en tiempos distintos y en lugares diferentes" (Dahl, 1999, p.12). Desarrolla el concepto de poliarquía como conjunto de reglas que designan cómo se llega al poder, quiénes llegan y cómo se ejerce. Tiene un conjunto de características y se corresponde con una manifestación empírica de las democracias contemporáneas.

Es importante destacar que la democracia como se viene mencionando, y la república se dividen en un momento específico en el cual se institucionaliza la república moderna como forma de gobierno representativo. Siéyès consideraba sobre todo la representación como la aplicación en el ámbito político de la división del trabajo, un principio que, en su opinión, constituye un factor clave del progreso social.

En relación a los impactos las tradiciones liberales y republicanas en el desarrollo conceptual de las instituciones de las democracias representativas modernas se puede hacer alusión a Ortiz Leroux (2014) porque considera que con el renacimiento de las ciudades luego de la Edad Media, el republicanismo ha tenido también un renacer, es decir, que el mismo no fue el resultado de la casualidad ni de una supuesta voluntad divina o ley histórica, sino el resultado, principalmente de un vacío o malestar que fue diagnosticado en el cuerpo político de las sociedades liberales contemporáneas. Es interesante considerar los aportes tanto de Roma como de Grecia Antigua en la constitución de lo que luego se dio a llamar democracias representativas.

En efecto, el Renacimiento de la teoría política republicana se ve relacionado a una crisis de doble entrada: por una parte, una crisis política ligada a la falta de representatividad y legalidad de las democracias liberales existentes, las cuales ha sustituido la figura política del ciudadano por una imagen económica del consumidor y "han relegado las virtudes cívicas clásicas por las virtudes institucionales modernas, y por otra parte una crisis teórica ligada a la fragilidad de los fundamentos normativos del liberalismo conservador" (Ortiz Leroux, 2014, p.24).

Por ejemplo, según Manin (2006) las repúblicas italianas de la Edad Media y del Renacimiento elegían a menudo sus magistrados por sorteo. En Florencia, centro intelectual del humanismo cívico y de la renovación republicana, la selección de magistrados por sorteo era una institución clave del sistema republicano.

El politólogo estadounidense, Dahl (1999), considera que la democracia es antigua y se pregunta “¿dónde se inició exactamente?”, algunos pueden pensar que la democracia comenzó hace doscientos años en los Estados Unidos, otros en la Grecia Antigua o Roma. La idea de una continuidad de la democracia lineal es una premisa que Dahl pone en cuestión por dos motivos. En primer lugar, porque después de los primeros siglos en Grecia y Roma, el progreso del gobierno 
popular derivó en su caída y desaparición. En segundo lugar, la democracia no fue inventada de una vez y para siempre. Aunque Roma, según Dahl (1999) ha sido un pueblo altamente creativo, nunca inventaron o adaptaron una solución que hoy no parece obvia: un sistema factible de gobierno representativo.

Para los Federalistas, "la constitución de una república moderna debe ser fundada en un conocimiento preciso de las consecuencias que tienen las leyes y de las instituciones en el comportamiento humano" (Ortiz Leroux, 2014, p.45). En palabras del autor, desde esta perspectiva las leyes e instituciones son las que aseguran la existencia y durabilidad de las repúblicas y no las cualidades cívicas de los ciudadanos.

La verdadera diferencia entre las antiguas democracias y las repúblicas modernas estriba, de acuerdo con Madison, en la «la absoluta exclusión del pueblo en su calidad de colectivo de cualquier participación en el gobierno en las segundas, y no en la absoluta exclusión de los representantes del pueblo de la administración en las primeras».

\begin{abstract}
Cabe recordar que, a diferencia del liberalismo, que es una teoría política moderna por definición, "república" es un concepto e institución antigua de la política que ha sobrevivido y se adaptado a las exigencias de la política moderna. De hecho, los valores, principios, tradiciones y lenguajes políticos republicanos remiten al mundo clásico grecolatino, pero el vocablo "republicanismo" surge a fines del siglo XVIII al agregarse el sufijo que implica movimiento. (Rodriguez Rial, 2019, p.57).
\end{abstract}

Para la autora desde el siglo XIX, la república empezó a ser usada como sinónimo de gobierno representativo. Pero esa república formal, moderna y liberal siempre resulta insuficiente. Para legitimarse políticamente el PRO (Propuesta Republicana, partido político argentino de centroderecha) apela a propuestas políticas más próximas al neo-republicanismo que al neoliberalismo cuando asume como propio en la campaña de 2015 el ideal de la inclusión (2019).

Para Bobbio, hoy no serían concebibles Estados liberales que no fuesen democráticos, ni Estados democráticos que no fuesen liberales. En suma, existen buenas razones para creer: a) que hoy el método democrático es necesario para salvaguardar los derechos fundamentales de la persona que son la base del Estado liberal; b) que la salvaguardia de estos derechos es necesaria para el funcionamiento correcto del método democrático. El liberalismo es una doctrina del Estado limitado tanto con respecto a sus poderes como a sus funciones.

La noción común que sirve para representar al primero es el estado de derecho; la noción común para representar el segundo es el estado mínimo. Aunque el liberalismo concibe al Estado tanto como estado de derecho cuanto, como estado mínimo, se puede dar un estado de derecho que no sea mínimo (por ejemplo, el estado social contemporáneo) y también se puede concebir un estado mínimo que no sea un estado de derecho (como el Leviatán hobbesiano ${ }^{1}$ respecto a la esfera económica que al mismo tiempo es absoluto en el más amplio sentido de la palabra y liberal en economía (Bobbio, 2005).

Manin (1998) considera que la democracia representativa permite a los ciudadanos dar la posibilidad de no elegir nuevamente a sus representantes en el caso que las promesas de campaña no se cumplan. El tema interesante en esta cuestión es la imposibilidad de saber si los

\footnotetext{
${ }^{1}$ Leviatán, o La materia, forma y poder de una república eclesiástica y civil, comúnmente llamado Leviatán, es el libro más conocido del filósofo político inglés Thomas Hobbes. Publicado en 1651, su título hace referencia al monstruo bíblico Leviatán, de poder descomunal.
} 
representantes que eligen en una elección de autoridades para el gobierno cumplan con lo estipulado.

No obstante, es significativo el hecho de poder no volver a votarlos y de este modo mostrar el desacuerdo por no cumplir. Es decir, que se pueden presentar promesas o programas, pero los representantes tienen la libertad de decidir cumplirlas o no. Sin embargo, cumplir las promesas, es una norma social que puede proveer el crecimiento político o seguir en el gobierno, no obstante, no cumplir acarrea dificultades a futuro en la reelección. Es por eso que según Manin, los gobiernos son sometidos regularmente a elección ya que para que los gobernantes sigan teniendo el privilegio y los beneficios que comporta estar gobernando se tienen que tener en cuenta los deseos del electorado cuando toman las decisiones.

\section{Estudio de caso: Presidencia de Mauricio Macri en Argentina (2015-2019)}

A modo de ejemplo, Macri es elegido a través del sistema democrático y representativo como presidente estando al frente del partido político Cambiemos (coalición política argentina con acuerdo con el PRO, UCR, Coalición Cívica ARI y otras fuerzas políticas) en el año 2015. Siguiendo a Manin (2006), la elección es, en efecto, una selección entre personas. Aunque los votantes comparen lo que dicen los candidatos, inevitablemente interviene la personalidad de los contendientes. Además, los programas y las promesas tienen una condición particular en los gobiernos representativos: no son vinculantes legalmente. Por el contrario, una vez elegida una persona, es ella quien decide la política pública.

"La doxa ${ }^{2}$ republicana" forma parte del discurso y de las estrategias políticas de legitimación de otros liderazgos de la "nueva derecha" que llegaron al gobierno, como se distingue en Cambiemos. Macri se consolida como figura política nacional y lidera la coalición Cambiemos, cuyos socios mayoritarios son Propuesta Republicana (PRO), la Unión Cívica Radical (UCR) y la Coalición Cívica como líder del partido político Cambiemos, teniendo también el compromiso de elegir su equipo. Más allá de la novedad que el PRO y su líder pretenden encarnar como un diferencial respecto de los partidos y dirigentes políticos más tradicionales, su trayectoria lo emparenta con la historia política argentina (Rodriguez Rial, 2019).

En ese sentido, es importante tener en cuenta el rol del Estado en primer lugar y cómo es planteado a través del gobierno de Mauricio Macri. Principalmente, cabe aclarar que el partido Cambiemos llega al poder por las elecciones nacionales y obligatorias a través del voto universal, secreto y obligatorio ya que la Argentina es un país que tiene un sistema de gobierno representativo, republicano, federal y democrático.

Las tendencias democráticas tienden a aparecer. Un avance hacia la participación democrática se desarrolla desde lo que se denomina: lógica de la igualdad (Dahl, 1999). Siguiendo a Canelo (2019), entre 2015 y 2019, el gobierno de Cambiemos no solo intentó desmantelar el modelo de sociedad implementado por los gobiernos kirchneristas desde 2003. Fue mucho más allá: puso en cuestión, con mayor o menor éxito, varios de los pactos, promesas y creencias que cimentaron la democracia de Argentina desde al menos 1983, y propuso reemplazarlos por otros nuevos o bien reciclados. Es decir, proponer otra forma de gobernar, con un modelo de país y de

\footnotetext{
2 Doxa es una palabra griega que se suele traducir por "opinión".
} 
Estado distinto al que se estaba representando con gobiernos anteriores al surgimiento de esta "nueva derecha democrática y moderna" (Natanson, 2018).

En relación a este régimen democrático argentino, el balance resulta complejo en lo concerniente a la dimensión de la rendición de cuentas horizontal. Si bien la participación efectiva y la competencia política evidencian una democracia, un enfoque multidimensional de la calidad democrática pone énfasis en otros factores, tales como la calidad de la vida social, el desarrollo de la ciudadanía, la eficacia institucional y, en forma particular, la rendición de cuentas horizontal (O’Donnell, Iazzetta y Vargas Cullel, 2003).

En ese sentido, Leiras (2018) considera según O’Donnell que adquiere especial relevancia para una adecuada comprensión de los diferentes niveles de responsabilidad -vertical y horizontal- a los cuales se encuentran sometidos los líderes políticos en su relación con la ciudadanía, las agencias de control institucional y los diferentes actores sociales y políticos.

El autor entiende la accountability horizontal (rendición de cuentas horizontal) como un concepto que delimita un tipo específico de interacción entre agencias estatales, más estrecho que el conjunto de controles e intercambios entre esas agencias. En todos los casos de accountability horizontal, una agencia estatal dada, directamente o a través de la movilización de otra agencia (a menudo un juzgado) se dirige a otra agencia (o agencias) estatal, sobre la base de argumentos legales basados, respecto de acciones (o inacciones) presumiblemente ilegales por parte de ésta.

La presidencia de Mauricio Macri resulta interesante analizar como un caso en donde las democracias delegativas surgen de las crisis.

Sus líderes movimientistas, portadores de la concepción hiperpresidencialista que resulta de su hostilidad a todo tipo de accountability, los lleva a presentarse y al parecer creerse auténticos "salvadores de la patria", quienes necesitan y merecen todos los poderes según ellos necesarios para rescatar a la patria. (Leiras, 2018, p. 80).

El balance del mandato de Macri resulta complejo en cuanto a la accountability horizontal ya que, por ejemplo, uno de los análisis que se puede realizar es en relación al menor nivel de producción legislativa; por otra, mayor iniciativa del Congreso, por momentos con una llamativa ausencia de trabajo político previo por parte del Ejecutivo nacional, aún en un contexto como el de las sesiones extraordinarias, previstas constitucionalmente para el tratamiento de la agenda propuesta por el poder Ejecutivo (Leiras, 2018).

En ese sentido, siguiendo al autor, la experiencia de Cambiemos marca el inicio de una nueva e incierta transición en la Argentina, no ya de gobierno sino de régimen democrático, de la democracia delegativa a una auténtica democracia representativa y republicana. Leiras (2018) tiene en cuenta los tres atributos centrales de la Democracia Delegativa para analizar el gobierno de Cambiemos.

La primera característica es que el proceso electoral en el que ha ganado la presidencia Mauricio Macri, se ha desarrollado bajo razonables condiciones de transparencia y competitividad, sin embargo, existieron denuncias del escrutinio en Provincia de Buenos Aires. Como segunda 
característica el terreno de las libertades civiles y políticas estaban razonablemente garantizadas aún en un contexto en donde se hablaba que había presos políticos del gobierno anterior.

En la última característica es donde Cambiemos ha marcado el inicio de una democracia republicana porque ha existido un funcionamiento de la Corte Suprema de Justicia que ha dado muestra de una independencia del poder Ejecutivo, también en un cambio en el poder legislativo porque el gobierno de Mauricio Macri era minoría en el congreso y se abrió otra nueva forma de relación entre el Poder Ejecutivo y el Poder Legislativo que ha mostrado una menor producción legislativa y mayor iniciativa del Congreso.

Existieron eventos para destacar, por ejemplo, el intento de designación de dos jueves de la Corte Suprema y la preservación de la Oficina Anticorrupción por parte de Cambiemos al designar a Laura Alonso a su cargo. Sin embargo, un informe brindado por la organización Open Knowledge International (OKI) ${ }^{3}$ del año 2016 ha ubicado a la Argentina en el puesto 20 del Índice Global de Datos Abiertos entre 94 países de todo el mundo en el ranking de transparencia y acceso a la información pública. En 2015 el país había ocupado la posición 54 entre 122, por lo que el año último subió 34 puestos, por lo que se convirtió en la nación de mayor crecimiento.

Según Leiras (2018), la particularidad del gobierno de Cambiemos, es que se trata de una experiencia, la primera en la República Argentina, en la que el presidente Mauricio Macri y líder de la organización Propuesta Republicana (PRO), no pertenece ni a la Unión Cívica Radical ni al peronismo (Partido Justicialista), dos de las expresiones más relevantes del Siglo XX. El discurso del líder de Cambiemos, Mauricio Macri, encuentra elementos constitutivos de republicanismo bélico del PRO cuya comprensión remite a la historia de las tradiciones de pensamiento político republicano.

\section{Conclusiones finales}

Para culminar el análisis propuesto, cabe recalcar que recuperar los debates clásicos sobre la Teoría de la democracia, dándole importancia a la historia de los conceptos políticos, permite poner en discusión el impacto de la democracia de los antiguos y de los modernos en la coyuntura política latinoamericana. También, un análisis desde esta perspectiva, posibilita poner en tensión si un gobierno representativo es lo mismo que representación, o repensar las relaciones que existen entre democracia y representación en un caso de liderazgo.

A lo largo de este escrito, se ha realizado un breve recorrido sobre el concepto de gobierno democrático hasta llegar al siglo XXI. La democracia está vinculada con la institucionalización y vigencia de derechos políticos de participación y deliberación siendo el más importante la participación del voto para llegar a una mayor igualdad. Recuperar su origen, sirve para repensar sucesos y hechos actuales. Es por ello que se trabaja con el significado de poliarquía, republicanismo, gobierno representativo, liberalismo, entre otros.

\footnotetext{
${ }^{3}$ La fundación Open Knowledge International (OKI) publicó su Índice global de apertura de datos 2016, disponible en https://index.okfn.org/place/ (consulta 31-01-2020)
} 
Trabajar con el caso de la presidencia de Mauricio Macri refleja, en primer lugar, que en la Argentina se están viviendo más de 30 años de democracia consecutivas, desde su recuperación en 1983. En ese sentido, la elección de un gobierno democrático ha sido a través del voto universal, secreto y obligatorio que dispone el país. Macri se consolidó como una figura política nacional, líder de la coalición Cambiemos. Su elección marcó el inicio de una democracia republicana, en contraposición al gobierno anterior. Resulta interesante y enriquecedor pensar no sólo las democracias delegativas, sino también el contexto en el que surgen los nuevos líderes políticos.

Las democracias actuales y también las históricas, no han podido prescindir de los liderazgos ni de las etapas fundantes ni de las transiciones. Es preciso remarcar que los liderazgos son actores de los procesos políticos democráticos, pero que tiene que vérselas y no siempre de la mejor manera.

\section{Bibliografía}

Bobbio, Norberto (2005). Liberalismo y democracia (México: Fondo de Cultura Económica), Cap. "La libertad de los antiguos y la libertad de los modernos", "La democracia de los antiguos y la de los modernos", "Liberales y democráticos en el siglo XIX", "Democracia representativa"

Dahl, Robert (1999). La democracia. Una guía para ciudadanos (Buenos Aires: Taurus). Cap. II “¿Dónde y cómo se inició el desarrollo de la democracia?"

Canelo, Paula (2019) ¿Cambiamos? La batalla cultural por el sentido común de los argentinos, Buenos Aires, XXI

García Linera, Álvaro (2008) "Crisis estatal y época de revolución”, en La potencia plebeya. Acción colectiva e identidades indígenas, obreras y populares en Bolivia, Buenos Aires: Prometeo/CLACSO.

Leiras, Santiago (2018). “¿Democracia delegativa? La experiencia de Cambiemos en Argentina (2015-2017)”Revista de Estudios Políticos y Estratégicos, 6 (1): 74-94, 2018 - ISSN 0719-3653 (impreso) ISSN 0719-3688 (en línea).

Malamud, A. (2019) “¿Se está muriendo la democracia? Nueva Sociedad No 282, julio-agosto 2019.

Manin, Bernard (2006). Los principios del Gobierno representativo (Madrid, Alianza), introducción, cap1, 2 y 5.

Murillo, V. (2019) “Democracia, intereses y estatus en América Latina” Nueva Sociedad No 282, julio-agosto 2019.

O`Donnell, G, Iazzetta, O. y Vargas Cullell, J. (2003). Democracia, desarrollo humano y ciudadanía. Reflexiones sobre la calidad de la democracia en América Latina. Rosario, Argentina: Homo Sapiens ediciones.

Ortiz Leroux, Sergio, (2014). República y republicanismo: una aproximación a sus itinerarios de vuelo" En defensa de la República. Lecciones de Teoría Política Republicana, (México, Editorial Coyoacán)

Rodriguez Rial (2019) La presencia de tradiciones y temas políticos clásicos en las nuevas derechas latinoamericanas: el republicanismo bélico del PRO. Estudios Sociales del Estado - volumen 5, número 9, pp. 55 a 80, primer semestre de 2019.

\section{Recursos electrónicos}

Índice global de apertura de datos en La fundación Open Knowledge International (OKI) (2016). Disponible en https://index.okfn.org/place/ (consulta 31-01-2020) 